\title{
de-Sitter space, (D)pp-Waves and star-Matrix Model
}

\author{
Harvendra Singh* \\ Theory Division, Saha Institute of Nuclear Physics, \\ 1-AF Bidhannagar, Kolkata 700064, India
}

\begin{abstract}
We consider de Sitter spacetime solutions and the corresponding de Sitter kind of plane-wave solution in $\mathrm{M}^{*}$ theory which is a $(2+9)$-dimensional theory. We attempt to write down corresponding matrix model which is found to have explicit negative energy mass terms as well as ghost-like kinetic terms for the matrix valued fields.
\end{abstract}

KeYwords: Penrose Limits, Plane-Waves, Matrix model.

*Formerly at: Department of Physics, Indian Institute of Technology, Guwahati, India 


\section{Contents}

1. Introduction 1

2. de-Sitter plane-waves (Dpp-wave) 2

2.1 The $d S_{5} \times H^{5}$ and a Dpp-wave solution 2

2.2 The time-like T-duality 3

3. A $\mathrm{D}(\mathrm{M}) \mathrm{pp}$-wave background in $\mathrm{M}^{*}$ theory

3.1 BMN* matrix model 5

3.2 Solutions 6

4. Conclusions 7

\section{Introduction}

The discovery of supersymmetric plane-wave ( or Hpp-wave) backgrounds in string theory [1, 2] has generated lot of interest in the string community in recent past. The reason behind this enthusiam has been again the hope of better understanding AdS/CFT correspondence [3]. The hint of this fact was demonstated by Blau et. al. [2] where it was shown that plane-wave backgrounds could simply be obtained by applying the Penrose limits [4, 5] on (anti)de Sitter spacetimes, $(A) d S \times$ Sphere. The plane-wave limits [2] basically make us zoom in onto those null geodesics which have a direction along sphere. Another fact is that string theory in pp-wave background becomes exactly solvable theory in suitable light-cone gauge [6, 7]. Therefore, from AdS/CFT correspondence point of view [3], a pp-wave spacetime in bulk has useful consequences for dual conformal field theories on the boundary 8 . Under this dictionary called BMN-correspondence [8], type IIB closed string excitations in a pp-wave background spacetime are dual to conformal field theory operators with large $U(1)$ R-charge. Since then there has been much progress in this field.

In this work we would like to concentrate on the plane-wave geometries in type II* string theories [9], which admit another kind of plane-waves known as de Sitter plane-waves (Dpp-wave) in [10]. These Dpp-waves can be obtained by taking ppwave limits of corresponding de Sitter spacetime solutions of type II* theories [10]. In this view, Dpp-waves can be as useful for dS/CFT holography [11 as are the supersymmetric Hpp-waves for AdS/CFT holography. 
It was proposed some time back by Hull [9] that if we consider time-like T-duality of usual type II string theory with spacetime signature $(1,9)$, then one can study string theories on arbitrary $(m, n)$ signature spacetimes. This duality along time direction somehow takes away the significance of the spacetime signature from the string theory, in particular the significance of Lorentzian symmetry $S O(1,9)$. However, while implementing time-like duality it requires the assumption of a compact time coordinate. It then makes the RR fields of the T-dual type II theory tachyonic (ghost-like) in nature, i.e., the kinetic terms with negative sign appear in the low energy supergravity action. These type II string theories in $(1+9)$ dimensions with tachyonic kinetic terms have been designated as $\mathrm{II}^{*}$ string theories [9]. Nevertheless, these star-theories are maximally supersymmetric string theories. We would like to note that the theories with tachyonic matter fields or with a tachyonic potential have been favourable candidates for cosmology, see [12] and references therein. The motivation is that we should be able to obtain de-Sitter spacetime solutions from string theory. The de Sitter spacetimes naturally appear as solutions in $\mathrm{II}^{*}$ theories. The usual string theory forbids de-Sitter space as a classical solutions, however see [13] for recent developments.

This paper is organised as follows. In section-2 we first review the $d S_{5} \times H^{5}$ de Sitter solution and corresponding Dpp-wave in type IIB* supergravity. Then we discuss time-like T-duality map between Hpp-wave and the Dpp-wave solutions. In section-3 we write down the analogous de Sitter plane-wave or $\mathrm{D}(\mathrm{M}) \mathrm{pp}$-wave solution in $(2+9)$ dimensional $\mathrm{M}^{*}$ theory. We then construct BMN-like matrix model which contains negative energy kinetic terms as well as negative energy mass terms for the bosonic fields. We find that fuzzy 2 -sphere solutions still form the ground state of the matrix model. The conclusions are given in section- 4 .

\section{2. de-Sitter plane-waves (Dpp-wave)}

\subsection{The $d S_{5} \times H^{5}$ and a Dpp-wave solution}

Consider type IIB* supergravity [9], the action has the kinetic terms for all the RR-potentials with negative signs. This theory can be obtained by implementing Tduality along compact time direction of ordinary type IIA theory. We shall assume that the II* theories are consistent string theories, when considered on a compact time-like circle, even though they have wrong sign kinetic terms of RR potentials in the low energy supergravity action. A quite remarkable property of these startheories is that, there do exist plenty of de-Sitter spacetime solutions in them, e.g. $d S_{5} \times H^{5}$ background in type IIB $^{*}$ theory [14, 15, 16]. As is shown in detail [14] ${ }^{1}$, these $d S_{5}$ vacua can be obtained by taking near horizon limits of Euclidean E4-

\footnotetext{
${ }^{1}$ I am thankful to Shibaji for bringing this work to my notice and for discussion.
} 
brane solutions of IIB* $^{*}$ theory. ${ }^{2}$ They are also maximally supersymmetric solutions. These E4-brane solutions are useful for dS/CFT holography, as are the D3-branes in AdS/CFT holography, for example. The corresponding dual super-Yang-Mills theory is defined in 4-Euclidean dimensions.

The de Sitter plane wave solutions can be obtained by considering Penrose-Güven limits of the $d S_{5} \times H^{5}$ background. These Dpp-wave solutions [10] obtained through Penrose limits are given by

$$
\begin{aligned}
& g:=2 d x^{+} d x^{-}+\left(\mu^{2} \delta_{a b} x^{a} x^{b}\right)\left(d x^{-}\right)^{2}+\sum_{a=1}^{8}\left(d x^{a}\right)^{2} \\
& F_{-1234}=2 \sqrt{2} \mu=F_{-5678}
\end{aligned}
$$

It can be seen that only nonvanishing componant of the Ricci tensor for the Dpp-wave metric (2.1) is $R_{--}=-8 \mu^{2}$, which is strictly negative. ${ }^{3}$ Also note that the coefficient of the $\left(d x^{-}\right)^{2}$ term in the Dpp-wave metric is positive definite which is opposite of the case of Hpp-wave [i]. To note Hpp-wave is a maximally supersymmetric pp-wave solution in type IIB theory. The reason for this is that the background (2.1) satisfies the field equations of type IIB* $^{*}$ supergravity where $C_{(4)}$ kinetic term has negative sign.

\subsection{The time-like T-duality}

We briefly discuss time-like duality map for the Dpp-wave solutions. The time-like T-duality map between the fields of two types of string theories has been constructed by Hull [9]. Under this map the background fields of type IIB (IIA) string theory are mapped to the fields of IIA* (IIB*) theory and vice-versa. We would like to show that Hpp-wave and the Dpp-wave are mapped to each other under the time-like Tduality along with space-like T-duality. Consider first the supersymmetric Hpp-wave solution of type IIB theory [1]

$$
\begin{aligned}
& g:=2 d x^{+} d x^{-}+\left(H_{a b} x^{a} x^{b}\right)\left(d x^{+}\right)^{2}+\sum_{a=1}^{8}\left(d x^{a}\right)^{2} \\
& F_{(5)}=2 \sqrt{2} \mu d x^{+}\left(d x^{1} d x^{2} d x^{3} d x^{4}+d x^{5} d x^{6} d x^{7} d x^{8}\right)
\end{aligned}
$$

with the matrix $H_{a b}=-\mu^{2} \delta_{a b}$. The Ricci tensor for Hpp-wave is $R_{++}=8 \mu^{2}$, which is positive unlike the Dpp-wave. In (2.2) we can consider $x^{+}$as time coordinate while

\footnotetext{
${ }^{2}$ Half-supersymmetric E $n$-branes in type II* theories have spacelike $n$ world-volume directions and $10-n$ transverse directions which include the time coordinate (with Dirichlet boundary conditions) 14 .

${ }^{3}$ We follow the convention where for a de Sitter space of radius of curvature $l$, the Ricci tensor is given by $R_{\mu \nu}=\frac{(D-1)}{l^{2}} g_{\mu \nu}$. Here $D$ is the spacetime dimension.
} 
$x^{-}$acts like a space-like direction. In this sense the 5-form flux is electric type. We can compactify $x^{+}$coordinate and make a time-like duality [9]. Using the duality relations, the background obtained as a result is extended string solution in type IIA* theory [10]. In subsequent step we make a space-like T-duality by compactifying the space-like string direction $x^{-}$of the string solution. That gives us a type IIB* plane wave solution [10]

$$
\begin{aligned}
& g:=2 d x^{+} d x^{-}+\left(\mu^{2} \delta_{a b} x^{a} x^{b}\right)\left(d x^{-}\right)^{2}+\sum_{a=1}^{8}\left(d x^{a}\right)^{2} \\
& F_{(5)}=2 \sqrt{2} \mu d x^{-}\left(d x^{1} d x^{2} d x^{3} d x^{4}+d x^{5} d x^{6} d x^{7} d x^{8}\right),
\end{aligned}
$$

which is nothing but the Dpp-wave as in (2.1). Since in (2.3) the coordinate $x^{-}$ behaves like a space direction, the flux $F_{(5)}$ is magnetic type. Hence the roles of $x^{+}$ and $x^{-}$have got exhanged under the above duality map which includes one time-like T-duality. However this is not of much significance because $x \rightarrow-x$ will exchange $x_{-} \leftrightarrow x_{+}$. The Dpp-waves are maximally supersymmetric as they are obtained from Penrose limits of the corresponding supersymmetric $d S_{5} \times H^{5}$ solutions [10]. Even otherwise the T-dualities preserve supersymmetry of the given background.

Thus we see that starting from Hpp-wave of IIB string and implementing a time-like duality and a space-like duality in succession, we have obtained Dpp-wave which is a solution of the IIB* string theory. In the next section we discuss de Sitter plane-wave solution in $\mathrm{M}^{*}$ theory. The motivation is that we should be able to study dS/CFT holography through Dpp-wave/CFT holography as in the BMN-model [ [8].

\section{A $\mathrm{D}(\mathrm{M}) \mathrm{pp}$-wave background in $\mathrm{M}^{*}$ theory}

As we know M-theory compactification on a square 2-torus, $T^{2}$, in the limit of shrinking torus size leads to type IIB string theory. Similarly, $\mathrm{M}^{*}$-theory compactification on a Lorentzian torus, $T^{1,1}$, in the limit of torus shrinking to zero area leads to type IIB* strings [9]. So it is natural to expect that the Dpp-wave like solutions in $\mathrm{M}^{*}$ theory. The $\mathrm{M}^{*}$ theory has $(2,9)$ spacetime signature. The supergravity action has the kinetic energy term for the 3 -form potential with negative sign and is given by [9]

$$
S=\int\left[\left\{R^{*} 1+\frac{1}{2} G_{(4)}{ }^{*} G_{(4)}\right\}+\frac{4}{3} d C_{(3)} d C_{(3)} C_{(3)}\right],
$$

The field strength is defined through $G_{(4)}=d C_{3}$. It is straight forward and obvious that there exists a Freund-Rubin type de-Sitter solution $d S_{(1,3)} \times H^{(6,1)}$ in $\mathrm{M}^{*}$ theory. This solution is rather interesting as it has four-dimensional de Sitter sub-spacetime and a seven-dimensional hyperbolic spacetime. The pp-wave limit of this de Sitter 
solution provides us with the following 11-dimensional plane-wave solution [2]

$$
\begin{aligned}
& g:=2 d x^{+} d x^{-}+H(x)\left(d x^{-}\right)^{2}+\eta_{a b} d x^{a} d x^{b} \\
& G_{(4)}=\mu d x^{-} d x^{1} d x^{2} d x^{3}
\end{aligned}
$$

where metric $\eta_{a b}=\operatorname{diag}(+, \cdots,+,-)$ is of indefinite signature involving one time like transverse direction and the function

$$
H=\frac{\mu^{2}}{9}\left[\left(x^{1}\right)^{2}+\cdots+\left(x^{3}\right)^{2}\right]+\frac{\mu^{2}}{36}\left[\left(x^{4}\right)^{2}+\cdots-\left(x^{9}\right)^{2}\right] .
$$

We will distinctly call the solution (3.2) as DMpp-wave. These DMpp-waves are endowed with noncompact rotational isometry group $S O(3) \times S O(5,1)$. This is quite unlike the usual supersymmetric (M)pp-wave of Matrix-theory [8], which has compact rotational isometry group $S O(3) \times S O(6)$ in the transverse directions. Also, $H(x)$ is indefinite here and specifically time dependent. We must note that timeindependent DMpp-waves can also be obtained, but we want to discuss most general case here.

\section{1 $\mathrm{BMN}^{*}$ matrix model}

Having obtained the eleven-dimensional DMpp-wave background in (3.2) it is imperative to discuss the BMN type matrix model [8] for this background. The BMN model [8] admits fuzzy 2-spheres and 5-branes as supersymmetric solutions. Let us assume $x^{+} \sim x^{+}+2 \pi R$, being periodic over a circle of radius and $R \equiv R_{B F S S}$ [17]. Then the dynamics of the theory in the momentum sector $p^{-}=p_{+}=N / R$ will be given by $U(N)$ matrix model. This can be understood in the following way.

As we know, $\mathrm{M}^{*}$ theory is considered as the (time-like) decompactification of type IIA* string theory in the strong coupling limit. At the same time analogue of D0-branes are the E1-branes in IIA* theory [14]. These supersymmetric $N$ E1-branes then will describe BFSS-like $U(N)$ matrix model

$$
S_{B F S S}^{\prime}=\int d t \operatorname{Tr}\left[\frac{1}{2 R}\left(D_{t} x^{a}\right)^{2}-\frac{R}{4}\left[x^{a}, x^{b}\right]^{2}+\text { fermionic terms }\right]
$$

where indices $a, b=1,2, \cdots, 9$ and time $t$ is identified with $x^{-} .4$ Indices $a, b$ are contracted with Minkowski metric $\eta_{a b}$. Comparing (3.3) to the BFSS matrix model [17], the kinetic term for field $x^{9}$ are with negative signs in (3.3), i.e. those are ghost like in nature. To note, the action (3.3) has been obtained by placing the Minkowskian metric $\eta_{a b}$ in the internal (rotational) space of the fields. Due to this the model has a noncompact rotational group symmetry $S O(1,8)$. The noncompact nature of isometry group $S O(1,8)$ is due to the fact that E1-branes, which give rise to matrix model dynamics at strong coupling, have one time direction and eight

\footnotetext{
${ }^{4}$ The time $t$ in this section must be viewed as Euclidean time.
} 
space directions with Dirichlet boundary conditions. ${ }^{5}$ The easiest way to ensure that all the signs are correct in the matrix model is to obtain it by compactifying ten-dimensional $S U(N)$ Super-Yang-Mills theory on $(1,8)$ torus.

Then corresponding to the DMpp-wave background, BMN like action can be written in analogous manner and we propose

$$
\begin{aligned}
& S=S_{B F S S}^{\prime}+S_{\text {mass }} \\
& S_{\text {mass }}=\int d t\left[\frac{1}{2 R} \operatorname{Tr}\left[\frac{\mu^{2}}{9}\left(x_{1}^{2}+\cdots+x_{3}^{2}\right)+\frac{\mu^{2}}{36}\left(x_{4}^{2}+\cdots-x_{9}^{2}\right)\right]-\frac{i \mu}{3} \epsilon_{r l s} \operatorname{Tr}\left(x^{r} x^{l} x^{s}\right)\right. \\
& + \text { fermionic terms }]
\end{aligned}
$$

where indices $a, b=1, \cdots, 9$; while $r, l=1,2,3$. Comparing (3.4) to the BMN matrix model [ 8 , the kinetic term for the field $x^{9}$ and its mass term appear with negative signs in (3.4), i.e. those are tachyonic in nature. The above action has been obtained by placing the Minkowskian metric $\eta_{a b}$ in the internal isometry (rotations) space of the fields $x^{a}$ in the BMN model and simultaneously changing the signs of all kinetic terms. However, we must note that it is now an Euclidean matrix model. The Myers term remains the same as in BMN model because the $G_{(4)}$ flux remains unchanged. The mass terms break the symmetry from $S O(1,8)$ to $S O(3) \times S O(5,1)$. If we set $\mu=0$ in the above $\mathrm{BMN}^{*}$ matrix model (3.4) we obtain BFSS-like matrix model (3.3), but one of the scalar field $x^{9}$ will be tachyonic owing to the noncompactness of the transverse isometry group. We have already mentioned, this is due to fact that we have E1-branes with time-like coordinate in the transverse space.

\subsection{Solutions}

We do find that the fuzzy 2-sphere is still a solution of the above matrix model (3.4). If we take

$$
\left[x_{r}, x_{l}\right]=i \frac{\mu}{3 R} \epsilon_{r l s} x_{s}
$$

with $\dot{x}^{a}=0, x^{4}=0=\cdots=x^{9}$, the classical equations of motion are satisfied. It will correspond to fuzzy sphere of physical radius in 1,2,3 space-like directions given by

$$
r \sim 2 \pi \sqrt{\frac{\operatorname{Tr} \sum_{r} x_{r}^{2}}{N}} \sim \pi \frac{\mu N}{3 R} .
$$

We cannot say anything about the supersymmetries preserved by this solutions. But, we expect them to be supersymmetric and stable.

The other interesting solutions are the fuzzy 2-torus type [18]. Consider a pair of $U(2)$ matrices $x^{4}, x^{5}$ we have a noncommuting solution

$$
x^{4} x^{5}=x^{5} x^{4} e^{i \theta}, \quad \theta \equiv \frac{2 \pi}{N}=\pi(\text { since } N=2)
$$

\footnotetext{
${ }^{5}$ The E1-branes though have tachyonic mass but become massless in the strong coupling limit, quite similar to the D0-branes becoming massless in matrix-model picture 14.
} 


$$
\left(x^{4}\right)^{2}=\left(x^{5}\right)^{2}=1, \quad\left(x^{4}\right)^{\dagger} x^{4}=1=\left(x^{5}\right)^{\dagger} x^{5}
$$

with $\dot{x}^{a}=0, x^{1}=x^{2}=x^{3}=0, x^{7}=0=\cdots=x^{9}$, provided mass parameter $\frac{\mu}{2 R}=12$. Such static solutions are possible for any pair of indices $a, b$ from the set of indices $4,5, \cdots, 9$. We can't again say definitely what would be the supersymmetry.

The time-dependent commuting configurations of the type

$$
\begin{aligned}
& x^{1}(t)=e^{-\frac{2 \mu}{3 R} t} x^{1}(0), \quad x^{2}(t)=e^{-\frac{2 \mu}{3 R} t} x^{2}(0) \\
& {\left[x^{1}(0), x^{2}(0)\right]=0}
\end{aligned}
$$

are also solutions. Similar time-dependent decaying solutions exist for any other pair of indices $a, b$ from $1,2,3$ or $4, \cdots, 9$.

\section{Conclusions}

We have studied de Sitter plane wave (or DMpp-wave) solutions in $\mathrm{M}^{*}$ theory in $(2,9)$ spacetime dimensions. These DMpp-waves are obtainable by employing the Penrose-Güven limits on the $d S_{(1,3)} \times H^{(6,1)}$ background of $\mathrm{M}^{*}$ theory. We find these DMpp-waves are quite analogous to maximally supersymmetric pp-wave geometry in M-theory, but have $S O(3) \times S O(5,1)$ non-compact rotational isometries. We have also written down BMN-like matrix model action for the DMpp-wave background. The matrix model allows the kinetic terms of fields with negative signs along with tachyonic mass terms. It is an expected feature because original low energy supergavity action for $\mathrm{M}^{*}$ theory has all these qualities [9]. Nevertheless it has been interesting to observe that the new $\mathrm{BMN}^{*}$ matrix model with tachyonic fields admits fuzzy 2-spheres as vacuum solutions which must be supersymmetric. We note that BMN model [8] has supersymmetric (stable) fuzzy 2-sphere vaccum solutions.

We would like to summarise by saying that it will be interesting to study further the $\mathrm{BMN}^{*}$ matrix model which quite nicely encodes the string (membrane) dynamics in the de Sitter backgrounds. Further we think that it will be worth while to understand the nature of Dpp-wave/CFT correspondence in type $\mathrm{II}^{*}$ string theories and the strong coupling picture through $\mathrm{BMN}^{*}$ matrix model, and hope that we may be able to understand the exact nature of dS/CFT correspondence [11].

\section{Acknowledgements}

I am grateful to Shibaji Roy for carefully reading the manuscript and for the comments. I am also thankful to the organisers of International Workshop on String Theory (ISM04) in Khajuraho for the invitation to present this work. The major part of this work has been carried out at Indian Institute of Technology, Guwahati. 


\section{References}

[1] M. Blau, J. Figueroa-O'Farrill, C. Hull and G. Papadopoulos, A new maximally supersymmetric background of IIB superstring theory, JHEP 0201 (2002) 047, hep-th/0110242.

[2] M. Blau, J. Figueroa-O'Farrill, C. Hull and G. Papadopoulos, Penrose limits and maximal supersymmetry, hep-th/0201081;

J. Figueroa-O'Farrill and G. Papadopoulos, Maximally Supersymmetric Solutions of Ten- and Eleven-dimensional Supergravities, hep-th/0211089.

[3] For a review see; O. Aharony, S.S. Gubser, J. Maldacena, H. Ooguri and Y. Oz, Phys. Rep. 323 (2000) 183, hep-th/9905111.

[4] R. Penrose, Any spacetime has a plane wave as a limit, in Differential Geometry and relativity, pp.271-75, Reidel, Dordrecht, 1976.

[5] R. Güven, Plane wave limits and T-duality, Phys. Lett. B 482 (2000) 255, hep-th/0005061.

[6] R.R. Matsaev, Type IIB Green-Schwarz superstring in plane wave Ramond-Ramond background, Nucl. Phys. B 625 (2002) 70, hep-th/0112044.

[7] R.R. Matsaev and A.A. Tseytlin, Exactly solvable model of superstring in plave wave Ramond-Ramond backgrond, hep-th/0202109.

[8] D. Berenstein, J. Maldacena and H. Nastase, Strings in flat space and pp wave from $N=4$ super Yang Mills, hep-th/0202021.

[9] C. Hull, Duality and the Signature of Space-Time, JHEP 9811, 017 (1998), hep-th/9807127.

[10] H. Singh, Plane wave type II* string backgrounds, Phys. Lett. B601 (2004) 209, hep-th/0405193.

[11] E. Witten, Quantum Gravity In De Sitter Space, hep-th/0106109; M. Spradlin, A. Strominger and A. Volovich, Les Houches Lectures on De Sitter Space, August 2001.

[12] A. Sen, Remarks on Techyon Driven Cosmology, hep-th/0312153 and references therein.

[13] S. Kachru, R. Kallosh, A. Linde and S. Trivedi, de Sitter vacua in string theory, Phys. Rev. D68 (2003) 046005, hep-th/0301240;

S. Kachru, R. Kallosh, A. Linde, J. Maldacena, L. McAllister and and S. Trivedi, Towards inflation in string theory, hep-th/0308055.

[14] C. Hull, JHEP 9807, 021 (1998), hep-th/9806146.

[15] G. Gibbons and C. Hull, De Sitter Space from Warped Supergravity Solutions, hep-th/0111072. 
[16] J.T. Liu, W.A. Sabra and W.Y. Wen, Consistent reductions of $I I B^{*} / M^{*}$ theory, JHEP 0401, 007 (2004) hep-th/0304253.

[17] T. Banks, W. Fischler, S.H. Shenker and L. Susskind, M-theory as a matrix model: A conjecture, Phys. Rev D55 (1997) 5112, hep-th/9610043.

[18] D. Biggati, Gauge Theory On The Fuzzy Torus, hep-th/0109018; J. Ambjorn, Y.M. Makeenko, J. Nishijima, R.J. Szabo, JHEP 0005 (2000) 023, hep-th/0004147. 